P\&A Año 3, N. ${ }^{\circ} 4$

enero-junio 2018

pp. [79]-91

\title{
Resumen
}

¿Cómo la arquitectura antroposófica responde y apoya a la pedagogía Waldorf? A lo largo del último siglo, se ha generado un mayor interés por parte de arquitectos, psicólogos y pedagogos acerca de la relación que existe entre arquitectura y educación, un interés por colocar al niño como eje del sistema educativo a quien se le debe brindar espacios de aprendizaje apropiados para su desarrollo. La pedagogía Waldorf, no solo ha transformado la forma de enseñar, además, ha generado una relación directa con la arquitectura del espacio de aprendizaje. En este contexto, el estudio de la arquitectura de dos colegios que implementan la pedagogía Waldorf de formas distintas en la ciudad de Lima permite entender cómo la arquitectura antroposófica contribuye al sistema pedagógico.

Palabras clave: Antroposofía, arquitectura antroposófica, arquitectura educativa, pedagogía no tradicional

\section{Arquitectura y antroposofía: La pedagogía Waldorf en los espacios de aprendizaje Colegio San Christoferus y Colegio Waldorf. Lima, Perú*}

\author{
Melany Vargas Benites*
}

Recibido: 8 de agosto de 2017

Aceptado: 18 de enero de 2018

\begin{abstract}
How does anthroposophical architecture respond and support Waldorf pedagogy? Over the last century, it has been generated a greater interest on architects, psychologists and pedagogues on the relationship between architecture and education, an interest in placing the child as the axis of the educational system and who must have appropriate space of learning for his development. Waldorf pedagogy has not only transformed the way of teaching but it has also generated a direct relationship with the architecture of the learning space. In this context, the study of the architecture of two schools that implement Waldorf pedagogy in different ways in the city of Lima, allows us to understand how anthroposophical architecture contributes to the pedagogical system.
\end{abstract}

Keywords: Anthroposophy, anthroposophical architecture, educational architecture, non-traditional pedagogy

* El presente artículo forma parte del trabajo de investigación realizado en el curso Taller de investigación dentro de la Facultad de Arquitectura y Urbanismo de la Pontificia Universidad Católica del Perú.

** Estudiante de la Facultad de Arquitectura y Urbanismo de la Pontificia Universidad Católica del Perú (PUCP). 


\section{Introducción}

El estado actual de las escuelas, tanto a nivel global como nacional, evidencian una resistencia de parte del sistema educativo a los cambios, lo que ha generado preocupación en un grupo de profesionales conformado por pedagogos, docentes, arquitectos, entre otros. Así, durante el siglo XX han surgido, en el campo de la educación, profesionales que han cuestionado el modelo de la escuela tradicional, desde el punto de vista de la arquitectura y los espacios de aprendizaje hasta la forma en cómo se organizan los cursos para cada grado. Pedagogos como Francesco Tonucci, Paulo Freire, María Montessori y Rudolf Steiner, se han pronunciado al respecto coincidiendo en que los espacios educativos no responden a las necesidades de los niños, ni a las nuevas formas en las que estos aprenden. Además, el desarrollo de nuevas tecnologías ha llevado a que se pueda acceder a la información con mayor facilidad, de la misma manera se ha logrado un entendimiento por parte de la población sobre la diversidad de los diferentes grupos de personas; sin embargo, ante estos cambios las escuelas tradicionales no han querido adaptarse y continúan trabajando de la misma manera que hace varios años atrás.

De esta manera, a lo largo del siglo XX se han propuesto nuevos sistemas educativos como la pedagogía Montessori, Waldorf y Reggio Emilia, que no solo proponen un cambio en la organización de los cursos, sino que además consideran a la arquitectura como un elemento fundamental para la enseñanza y el desarrollo de los niños. La arquitectura para estas escuelas alternativas es considerada un maestro más, también puede decirse que la arquitectura es vista como una envoltura dentro de la cual el niño podrá aprender, y podrán configurarse como espacios flexibles que se adaptan a las necesidades de los niños, asimismo, es en estas nuevas escuelas que el niño deja de ser solo un receptor de información y pasa a ser un elemento principal en el sistema educativo remplazando al maestro.

En el caso de las escuelas en el Perú, aún pueden encontrarse espacios obsoletos que se siguen utilizando para la enseñanza, edificios construidos recientemente, pero que siguen presentando las mismas características que aquellos construidos en décadas pasadas, espacios rectangulares donde los alumnos solo van a recibir información y docentes que solo enseñan, colegios que necesitan ser reemplazados por problemas estructurales, grandes brechas entre el número de instituciones educativas y el número de estudiantes por cada una de estas y la brecha de infraestructura educativa que asciende a cien mil millones de soles. El resultado del último Censo educativo realizado en el país en el año 2017 muestra que el total de alumnos matriculados en educación básica regular es de 7731 939 de los cuales 1936922 pertenecen a instituciones privadas. Por otro lado, la cifra a nivel nacional de escuelas públicas es de 82 018 y la cifra de escuelas privadas es de 24 558, haciendo un total de 106576 escuela. A pesar de que no existe una gran diferencia en la densidad de estudiantes, según la cantidad de escuelas, alrededor del $40 \%$ de las instituciones educativas públicas a nivel nacional requieren ser reemplazadas por problemas estructurales, deterioro o porque ya cumplieron su periodo de vida, obligando así a un porcentaje de niños a estudiar en espacios que no son adecuados.

La situación de la infraestructura educativa y la tasa neta de matrícula en Lima metropolitana, según Lima como vamos, ha incrementado en los niveles de inicial y secundaria, en el caso del nivel de primaria la variable se mantiene. En cuanto a la percepción de la calidad educativa solo el $19 \%$ de los encuestados se encuentra satisfecho con los servicios educativos; sin embargo, si este porcentaje se analiza desde los diferentes sectores socioeconómicos la realidad cambia, el $21 \%$ de personas que se encuentra satisfecha pertenece al sector $\mathrm{A} / \mathrm{B}$.

Ante este panorama han surgido nuevas propuestas educativas en la ciudad de Lima. Las primeras escuelas con metodología no tradicional llegan a la ciudad a finales del siglo $\mathrm{XX}$, así la primera escuela Waldorf se construye en el año 1982 en un terreno de casi $20000 \mathrm{~m}^{2}$, rompiendo así con los ideales de 
las escuelas tradicionales que se daban hasta el momento, para proponer nuevos espacios de aprendizaje que toman en cuenta las necesidades de los niños no solo a nivel intelectual, para adaptarse a los paradigmas del momento. Como parte de la arquitectura antroposófica, las aulas, son concebidas a partir de formas orgánicas que permiten que la clase se convierta en un elemento flexible según los requerimientos de cada curso. Así, se rompe el principio de las escuelas tradicionales en el Perú dando paso a la llegada de nuevos sistemas pedagógicos.

De la misma manera, entre finales del siglo $\mathrm{XX}$ y comienzos del siglo XXI, surgen otros modelos educativos en el Perú que se oponen al sistema tradicional; sin embargo, muchas de estas escuelas solo responden a la metodología mas no a un tema arquitectónico, en la mayoría de casos son casas o edificios que han sido adaptados para que de cierta forma puedan funcionar como espacios de aprendizaje. A pesar de los casos de escuelas con nuevas pedagogías que solo adaptan el espacio, en los últimos años se han desarrollado nuevos proyectos de arquitectura educativa que buscan generar los espacios adecuados para el aprendizaje de los niños.

Se necesita que la arquitectura nazca desde una forma de pensamiento pedagógico y que la pedagogía tenga en cuenta la experiencia vital del espacio arquitectónico, aceptando como reto un compromiso propositivo de transformación de la realidad que deseamos asumir (Cabanellas y Eslava, 2005, p.172).

Así, se entiende que la relación entre la arquitectura y la enseñanza son factores indispensables para los nuevos espacios de aprendizaje propuestos por las nuevas pedagogías, que consideran al espacio como un maestro que debe acompañar al niño durante su crecimiento y este debe transformarse a medida que el niño lo haga, considerando el espacio tan importante como lo es el profesor.

El análisis de dos colegios con pedagogía Waldorf en la ciudad de Lima, que se encuentran insertados en realidades diferentes y están orientados a niños con diferentes habilidades, permite entender cómo la arquitectura antroposófica contribuye al sistema pedagógico y cómo estos espacios influyen en el desarrollo del aprendizaje del niño. Los análisis arquitectónicos de ambos casos tienen en cuenta conceptos de antroposofía y arquitectura antroposófica.

\section{Pedagogía Waldorf: un movimiento derivado de la antroposofía}

La pedagogía Waldorf, movimiento derivado de la antroposofía, fue creada por el pedagogo, filósofo y arquitecto austríaco Rudolf Steiner. La primera escuela se fundada en el año 1919 en Stuttgart, Alemania, para la educación de los hijos de los trabajadores de la fábrica de cigarrillos Waldorf-Astoria, quienes buscaban una alternativa pedagógica diferente a la que se estaba dando en ese momento. De esta manera, "Lo que todos los colegios Waldorf tienen en común es un enfoque educacional que está radicalmente centrado en el niño y basado en el continuo estudio del ser humano en el desarrollo" (Clouder y Rawson, 2002, p.21), así Steiner propone una nueva escuela donde colocaba al niño como eje principal del sistema educativo

\section{La pedagogía Waldorf y su respuesta en la arquitectura}

A partir del análisis del libro de Kenneth Bayes, Arquitectura Viva, se extraen conceptos que muestran la relación entre la pedagogía, la arquitectura y la antroposofía. Así, conceptos como: arquitectura orgánica, arquitectura bioclimática, agricultura biológica y bioconstrucción son conceptos utilizados para generar ambientes que fomentan el desarrollo de las habilidades de los niños dentro y en los espacios exteriores de las escuelas.

Por otro lado, la pedagogía de la escuela Waldorf, al estar basada en los estudios sobre antroposofía y el concepto de ciencia espiritual de Rudolf Steiner, tiene como objetivo principal lograr el desarrollo integral del niño contemplando todo su ser, mente, cuerpo y alma. Para alcanzar este objetivo se modificó la forma de enseñanza de la pedagogía tradicional, 
como menciona Jiménez (2014): "la separación de tres conjuntos bien diferenciados está justificada por la concepción de septenios. Estos organizan y separan la enseñanza según la edad" (p.113); así, la pedagogía Waldorf se adecúa a las características y necesidades propias de cada grupo etario. El estudio de la división por etapas durante el desarrollo del hombre permite entender que cada grupo etario posee diferentes necesidades y por lo tanto la arquitectura debe responder a estos a través de diferentes configuraciones espaciales del aula.

\section{Arquitectura orgánica}

El concepto de arquitectura orgánica, de acuerdo a Jiménez (2009), busca que las formas evolucionen como lo haría un ser vivo, lo cual implica crecimiento, desarrollo y movimiento. Además, la arquitectura orgánica siempre brindará una experiencia sensorial al usuario a través de las formas libres (Bayes, 2014). De esta forma, los salones de la escuela son pensados como espacios flexibles que no solo permiten que las energías de los niños fluyan a través de las formas orgánicas, sino que dan la posibilidad de que el aula se transforme de acuerdo a las actividades que se realizan en el interior. La arquitectura antroposófica evita el uso de ángulos rectos y adopta las formas orgánicas tanto en planta como en elevaciones y secciones, de esta manera las formas libres crean un diálogo constante entre los techos y los muros, al igual que la antroposofía busca un diálogo entre las partes y el todo.

\section{Arquitectura bioclimática y bioconstrucción}

El concepto de arquitectura bioclimática busca que la arquitectura de las escuelas Waldorf se adapten al entorno y al lugar en el que se encuentran, estudiar las condiciones climáticas antes de proyectar el edificio es fundamental ya que se busca el equilibrio y armonía constante entre el hombre, el edificio y la naturaleza. Las escuelas Waldorf a través del concepto de arquitectura bioclimática buscan generar un edificio que es capaz de captar la mayor luz natural posible, así se contribuye al cuidado del medio ambiente y a reducir los costos de energía.

Por otro lado, entendiendo que los niños aprenden a través de las experiencias sensoriales, los espacios de una escuela Waldorf deben invitar a los niños a la experimentación y descubrimiento de nueva información a partir de lo observado, así, lo primero que se percibe al ingresar a una escuela Waldorf es el uso de diferentes materiales y colores, los que despiertan cada uno de los sentidos del niño. Además, Steiner analizó el efecto del color en la percepción y la conducta humana, basándose en las teorías del color de Goethe. Si bien no todas las escuelas son iguales en su selección de colores, el uso de este se basa en una respuesta a las etapas del desarrollo infantil. Los colores de las aulas evolucionan según el grupo de edad, el tono rosa se convertirá a un color amarillo alrededor del tercer grado, hasta los verdes para cuarto y quinto grado, es en este punto de la infancia donde se logra un equilibrio antes de la pubertad. En los últimos grados de secundaria los tonos azules y violetas serán los indicados para el trabajo reflexivo de los adolescentes.

\section{Pedagogía Waldorf en la ciudad de Lima}

El proceso de formación del primer colegio Waldorf en Lima se da en un período de tiempo largo, ya que estos dependen de la contribución de un grupo de personas, entre padres y maestros, quienes buscan brindar nuevos espacios de aprendizaje con características que rompan con los ideales de la pedagogía tradicional. Así, a mediados de 1980 se funda el primer jardín de infancia con pedagogía Waldorf para los hijos de los trabajadores de la fábrica Hidrostal ubicada en el distrito de Zárate, conservando el esquema de la primera escuela Waldorf alemana. Este jardín de infancia puede ser considerado como un detonador que llevó al diseño e implementación de nuevas escuelas Waldorf en Lima. A lo largo de cuarenta años se han formado en total nueve centros con la pedagogía Waldorf en Lima, todos ubicados en distritos con diferentes realidades, algunos responden a más de un concepto de arquitectura antroposófi- 
ca, otros abarcan los conceptos que no significan invertir mucho dinero, pero finalmente el objetivo de todos es brindar espacios que respondan a las actividades particulares que se llevan a cabo dentro de estos colegios.

\section{Colegio Waldorf-Lima}

Ubicado en el distrito de La Molina, el colegio Waldorf-Lima fue fundado en el año 1982, siendo así el primer colegio no tradicional Waldorf en el Perú. El programa educativo, hasta ese momento, solo había planteado el nivel de kindergarten y los tres primeros niveles de educación; años más tarde, por la acogida del nuevo sistema de enseñanza se propuso ser un colegio integral, así en el año 1988 se implementó la enseñanza hasta el nivel secundaria (Figura 1).

\section{El lugar}

El colegio Waldorf-Lima se encuentra ubicado en el distrito de La Molina. Según datos del Ministerio de Educación, el distrito de la Molina cuenta con un total de 69 colegios, de los cuales solo 9 son colegios públicos y 60 son colegios privados, brindando así educa- ción a un número aproximado de 15555 niños y adolescentes. Por otro lado, la cifra de colegios que utilizan un sistema de pedagogía no tradicional se reduce solo a uno, siendo este el colegio Waldorf.

Emplazado dentro de la urbanización Parque de Monterrico, el colegio cuenta con tres frentes: la Vía de Evitamiento, que se encuentra en el lado noroeste, la avenida Las Palmeras, que se encuentra en el lado sur y la calle José Antonio, hacia el este. La zona en la que se encuentra está conformada por una serie de centros de educación básica regular privados, tales como el colegio Peruano Norteamericano Abraham Lincoln, el colegio Santa María Eufrasia y el colegio Roosevelt, además de un solo centro de educación pública.

El terreno utilizado por el colegio Waldorf cuenta con un área de $21000 \mathrm{~m}^{2}$ de los cuales $3900 \mathrm{~m}^{2}$ representa el área techada, dejando así casi $85 \%$ de área libre. Como se puede apreciar en el plano, el área verde libre se encuentra dividida en nueve jardines, estos tienen usos como biohuerto, área para juegos de los estudiantes, canchas de deporte, etc.,

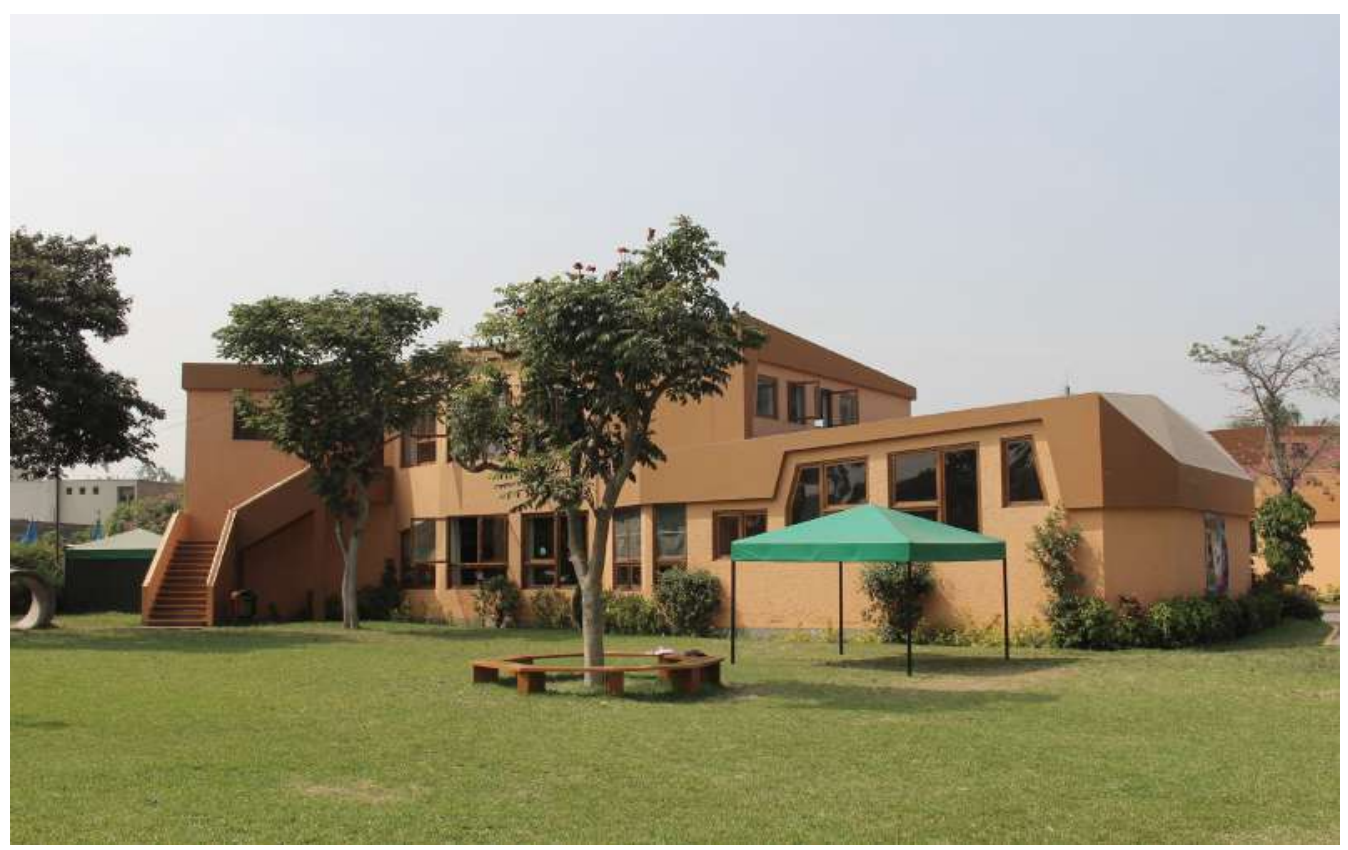

Figura 1. Vista desde el patio interior hacia el edificio de aulas del colegio Waldorf-Lima. (Fotografía: Vargas, M. 2017). 
con lo cual el colegio se plantea como un gran espacio de área verde dentro del distrito de La Molina. Por otro lado, el edificio principal en donde se encuentran las aulas, se emplaza en el lado este del terreno, mientras que la zona noroeste, que se encuentra más cerca a la vía de Evitamiento, funciona como una barrera que amortigua el ruido del alto tránsito vehicular.

Por otro lado, según Kenneth Bayes (2014), Steiner tenía un fuerte sentido de "lugar" y consideraba que el edificio debía tomar formas apropiadas dependiendo del sitio en el que este se encuentre, si estaba dentro de la ciudad o en el campo. Asimismo, consideraba a el edificio como un intermediario entre la tierra y el cielo. Para la antroposofía es fundamental tener en cuenta los criterios de arquitectura bioclimática; elementos como la ventilación y la luz natural son utilizados para el diseño de los espacios de aprendizaje ya que, como se mencionó anteriormente, existe una relación directa entre la naturaleza y los principios antroposóficos, donde se reconoce a la tierra como un ser vivo y como tal debe existir un equilibrio entre el edificio que se proyecta y el lugar que se está dañando al momento de hacer arquitectura; así, lo que se busca es utilizar criterios de diseño que se integren a las condiciones climáticas de la ciudad de Lima para evitar el uso de tecnologías que sigan dañando el ecosistema.

Es por esta razón que la disposición de las aulas responde a las condicionantes climáticas. recorrido solar $\mathrm{v}$ ventilación. Las aulas del nivel de primaria y secundaria se disponen con orientación norte-sur, estas recibirán una iluminación indirecta durante las mañanas de verano (Figura 2), mientras que las aulas orientadas hacia el norte recibirán los rayos solares directos, hasta el mediodía, durante el invierno; sin embargo, la cubierta ha sido diseñada para que los rayos solares no afecten los espacios de enseñanza (Figura 3). En el caso del edificio de inicial, durante las horas de la tarde, en verano y otoño, la iluminación indirecta penetrará por las ventanas, bañando así los espacios de aprendizaje con una luz natural que creará una atmósfera adecuada para los niños.

\section{El cuerpo en el espacio}

La forma del colegio Waldorf, de acuerdo a la arquitectura antroposófica, posee criterios de arquitectura orgánica, estos no solo responden a un concepto formal de forma y función. Según Jiménez (2009), el espacio debe funcionar como un medio para acompañar al niño no solo durante su etapa de desarrollo a nivel intelectual sino también a nivel anímico y espiritual. El colegio está conformado por seis edificios: vivienda del administrador, vivienda de maestros, cafetería, jardín de infancia, edificio de aulas, carpintería y depósito de jardinería, los cuales se encuentran organizados como elementos sueltos envueltos por área verde.

El ingreso al edificio principal se encuentra en la Av. Tosé Antonio. este edificio está

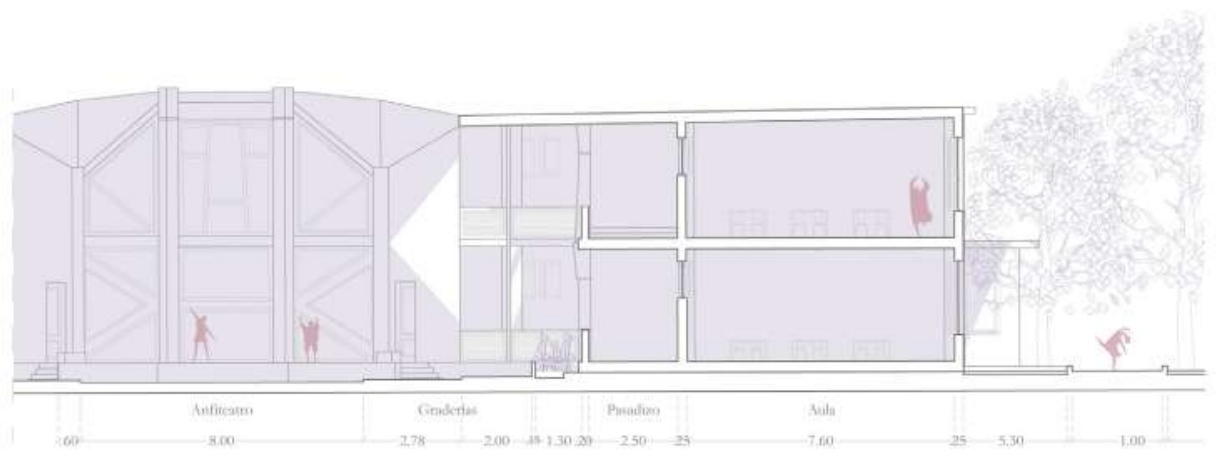

Figura 2. Análisis solar durante verano en el colegio Waldorf-Lima. (Elaboración: Vargas, M. 2017). 


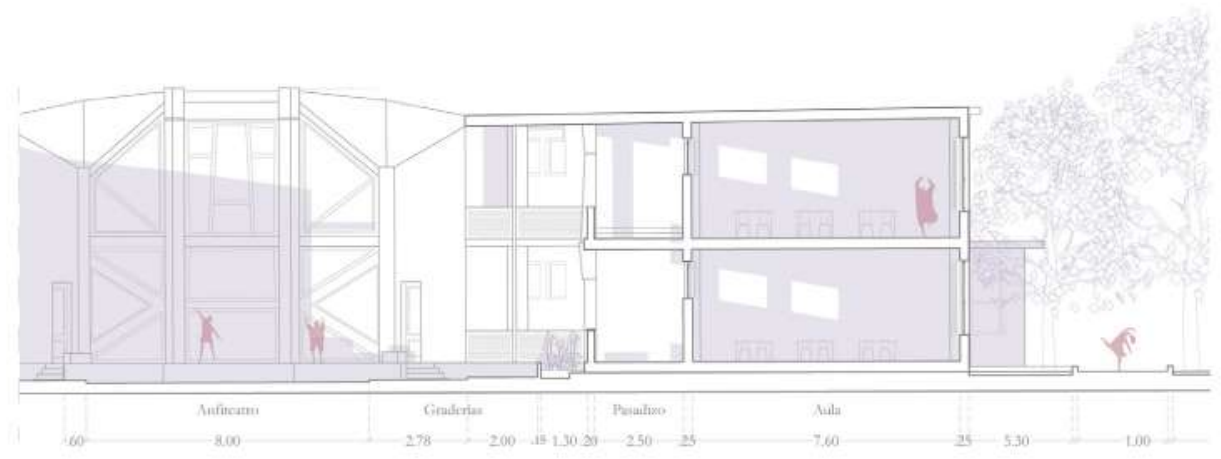

Figura 3. Análisis solar durante invierno en el colegio Waldorf-Lima. (Elaboración: Vargas, M. 2017).

conformado en el primer nivel por seis aulas que corresponden a los seis primeros grados de primaria, un aula de gimnasia, un aula de física, la sala de euritmia, la biblioteca y el área de profesores; en el segundo nivel se encuentran las aulas del nivel secundaria y un aula de idiomas. El edificio ha sido diseñado a partir de los criterios de arquitectura orgánica, tanto en el exterior como en el interior, además la arquitectura antroposófica aplicada a las escuelas Waldorf busca que todos los espacios respondan a un propósito en específico, además estos espacios deben integrarse y relacionarse generando un diálogo constante entre las partes y el todo. En el colegio Waldorf se puede apreciar este concepto de diseño en cómo han sido proyectadas las aulas de clase en relación al espacio exterior principal, la disposición de las aulas, tanto de primaria como de secundaria, se extienden alrededor de un espacio central llegándolo a "abrazar". Es en este espacio central exterior donde se coloca el anfiteatro (Figura 4).

Por otro lado, las aulas de clase desde el primer grado de primaria hasta el onceavo grado son rectangulares y ligeramente diferentes, a pesar de que Jiménez (2009) menciona que la forma del espacio de enseñanza debe evolucionar según el crecimiento del niño buscando formas orgánicas mientras estén en los primeros grados y evolucionen a formas más rígidas en los últimos años, en el colegio Waldorf-Lima esto no sucede; sin embargo, sí se ha tenido en cuenta que los espacios no sean del todo rectangulares evitando muros paralelos y generando un quiebre en uno de sus lados. Además, todas las aulas se encuentran conectadas por un pasadizo que da hacia el anfiteatro, generando una relación directa con el exterior.

En cuanto al uso de color en los espacios de aprendizaje del colegio Waldorf-Lima, esta se encuentra presente en todo momento. El exterior del edificio principal se encuentra pintado en tonos naranjas, mientras que en el interior las diferentes gamas de colores van cambiando de acuerdo al grado de estudio. Así, en el nivel de jardín de infancia todo el interior presenta tonalidades que van de los rojos a los rosados, esto responde a la teoría de Steiner en la que afirma que el niño pequeño al estar en contacto con estas tonalidades rojas estas se proyectan en su interior transformándolas en su color complementario, en este caso el verde color asociado a la tranquilidad, de esta manera a través del color se logra controlar el temperamento del niño inhibiendo el exceso de energía.

De la misma manera, en el edificio de aulas de primaria y secundaria, las aulas de clase están pintadas en tonos que irán degradándose desde los naranjas para los primeros grados hasta perder las tonalidades rojas para convertirse en azules y azules violetas para los últimos grados de estudio donde lo que se busca es que el adolescente comience a desarrollar su nivel de pensamiento, así se utilizan los azules para lograr un nivel de concen- 

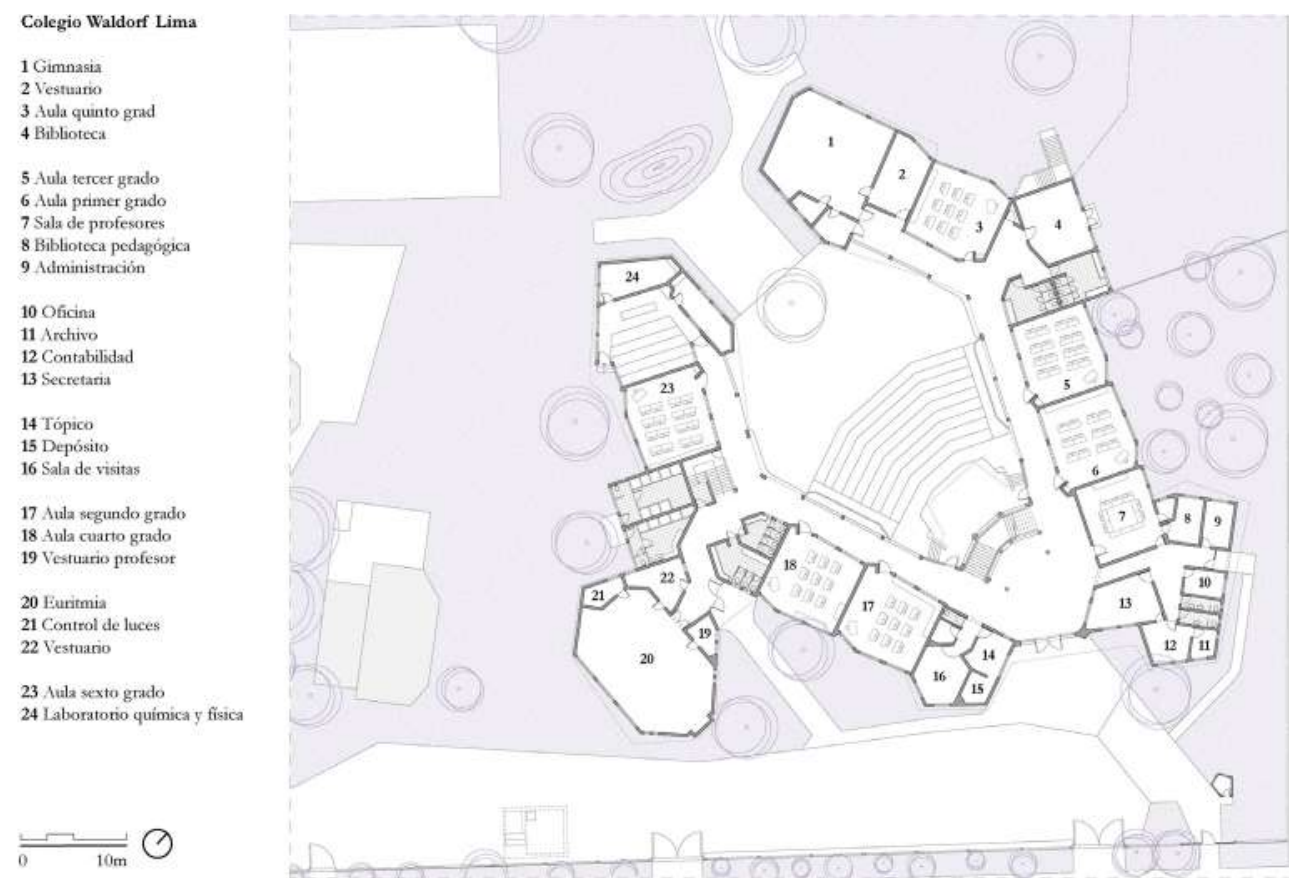

Figura 4. Distribución de espacios en el colegio Waldorf-Lima. (Elaboración: Vargas, M. 2017).

tración que los lleve a alcanzar sus objetivos. Cabe mencionar, que el único espacio dentro del edificio de aulas que no ha sido pintando de un color en específico es el laboratorio de física y química esto debido a que lo que se busca dentro del laboratorio no es controlar el temperamento de los niños.

\section{Colegio de educación especial San Christoferus}

Ubicado en el distrito de Chorrillos, el colegio de educación especial San Christoferus fue fundado en el año 1986. Nace a partir de la necesidad de generar un espacio que responda a las necesidades de niños con distintos tipos de habilidades diferentes. Es la única escuela Waldorf en el Perú que utiliza la pedagogía curativa antroposófica para ayudar al desarrollo de los niños. En la actualidad, el tema de la pedagogía Waldorf relacionada con la enseñanza de niños con habilidades diferentes no ha sido investigada a fondo; sin embargo, existen estudios que realizó Rudolf Steiner sobre la medicina antroposófica y cómo esta puede ser implementada en la enseñanza y en la arquitectura.

En este sentido, la pedagogía curativa antroposófica contempla al niño desde una forma holística entendiendo que, a pesar de los retrasos en el desarrollo del niño, ya sea a nivel físico o intelectual que se han podido generar, se busca respetar su desarrollo natural y además se trata de liberar a la persona sana que se encuentra detrás de todos los síntomas físicos e intelectuales.

"Los niños con discapacidades experimentan la mayoría de las veces, sus limitaciones e impedimentos, más rápido y con más fuerza, que otros niños. El estímulo y apoyo necesario para superarlos se concentra en las posibilidades terapéuticas." (Grimm, s.f., p. 5). Así, para apoyar al niño en su desarrollo se implementan una serie de talleres como el de carpintería, manualidades, panadería, además del arte de la euritmia, implementado en todos los colegios Waldorf, a partir de estas actividades se espera que los niños logren desarrollarse a nivel anímico y espiritual. 


\section{El lugar}

El colegio San Christoferus se encuentra ubicado en el distrito de Chorrillos. Según datos del Ministerio de Educación, el distrito de Chorrillos cuenta con un total de 170 colegios, de los cuales 31 son colegios públicos y 139 son colegios privados, además dentro del distrito de Chorrillos se encuentran 5 centro de educación básica especial, brindando así educación a un número aproximado de 32 900 niños y adolescentes. Por otro lado, la cifra de colegios que utilizan un sistema de pedagogía no tradicional curativa se reduce solo a uno, siendo este el colegio San Christoferus.

Emplazado en la urbanización La Campiña dentro de una zona de viviendas de densidad baja de uso mixto tanto viviendas-taller como viviendas-comercio, hacia el norte El Colegio San Christoferus limita con el Colegio Innova Schools y con la tienda Promart, por el este limita con el Centro Comercial Real Plaza y por el oeste limita con viviendas, fábricas y almacenes. Cuenta con un área de $12532 \mathrm{~m}^{2}$ de los cuales $1791 \mathrm{~m}^{2}$ representa el área techada, dejando así casi $87 \%$ de área libre la cual es utilizada como un biohuerto y área para juegos de los alumnos. De esta manera el colegio se plantea como un gran espacio de área verde dentro de una zona industrial.
La forma del terreno fue tomada en cuenta al momento del diseño del patio de juegos, además la transición que se genera desde el ingreso al colegio hasta llegar al edificio principal donde se encuentran las aulas y el espacio para la euritmia, conlleva una gran complejidad. Se ha planteado un camino que conduce al edificio, desde la puerta de ingreso principal hasta el ingreso del segundo pabellón, este camino oscilante atraviesa el patio de juegos y el biohuerto, además la zona posterior del pabellón de aulas se compone de una serie de caminos sobre el área verde que conecta este edificio con los salones de panadería, los hornos artesanales y el salón de carpintería.

Para la antroposofía es fundamental tener en cuenta los criterios de arquitectura bioclimática, elementos como la ventilación y la luz natural son utilizados para el diseño de los espacios de aprendizaje. Las aulas se disponen con orientación norte-sur, de esta manera los rayos solares directos no llegan al interior del espacio de trabajo de los niños durante las primeras horas del día, esto permite generar una atmósfera adecuada para realizar actividades en el interior (Figura 6). Las dos aulas que se enfrentan al este son protegidas por la cubierta, la cual ha sido diseñada para que los rayos solares no ingresen directamente en

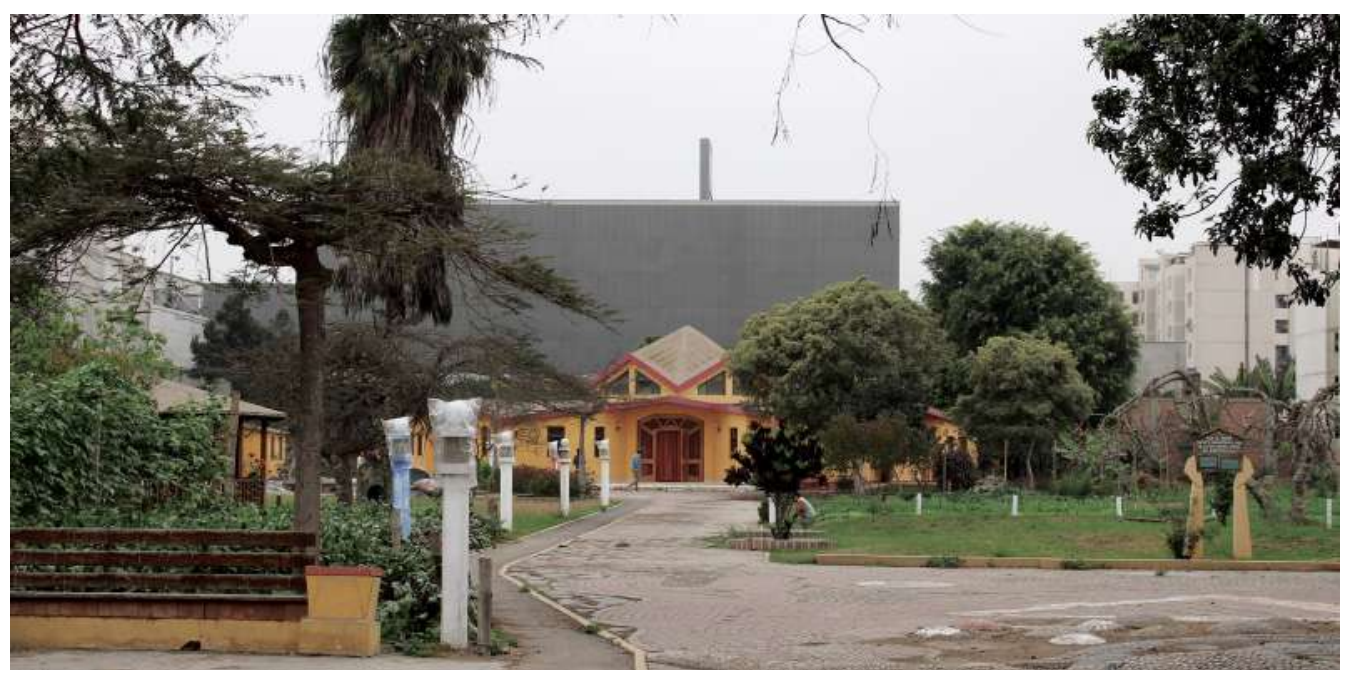

Figura 5. Vista del edificio de aulas y huerto del colegio de educación especial San Christoferus. (Fotografía: Vargas, M. 2017). 


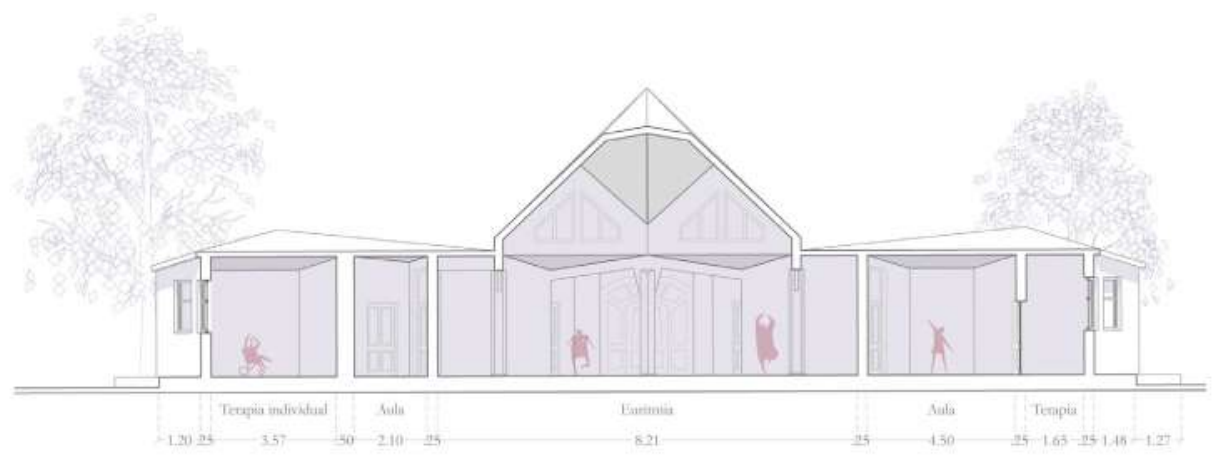

Figura 6: Análisis solar durante verano en el colegio Waldorf-Lima. (Elaboración: Vargas, M. 2017).

el espacio. Además, durante las horas de la tarde la iluminación directa penetra por las ventanas que se ubican en la cubierta, bañando así el espacio principal de la euritmia con una luz natural que irá cambiando según las estaciones. De esta manera, la iluminación natural ha sido un elemento fundamental a la hora del diseño del edificio para asegurar el ingreso de luz natural a través de las grandes ventanas de las aulas durante el día (Figura 7).

\section{El cuerpo en el espacio}

La forma del edificio, de acuerdo a la arquitectura antroposófica, posee criterios de arquitectura orgánica, estos no solo responden a un concepto formal de forma y función. Según Jiménez (2009), el espacio debe funcionar como un medio para acompañar al niño no solo durante su etapa de desarrollo a nivel intelectual sino también a nivel anímico y espiritual. Para el diseño del colegio San Christoferus se han tenido en cuenta las necesidades de los niños, quienes al tener algún tipo de discapacidad en menor o mayor nivel, necesitaban de un tipo de cuidados específicos, por lo cual, además de tomar en cuenta conceptos de la medicina antroposófica, se optó por tomar en cuenta el concepto de arquitectura orgánica.

El Colegio San Christoferus está compuesto por nueve edificios, de los cuales dos están conformados por aulas y los otros siete sirven como un apoyo a estos edificios. Así, también se encuentra dentro del terreno un edificio de viviendas para un par de familias de los niños que pertenecen a la institución, una pequeña vivienda para el encargado del cole-

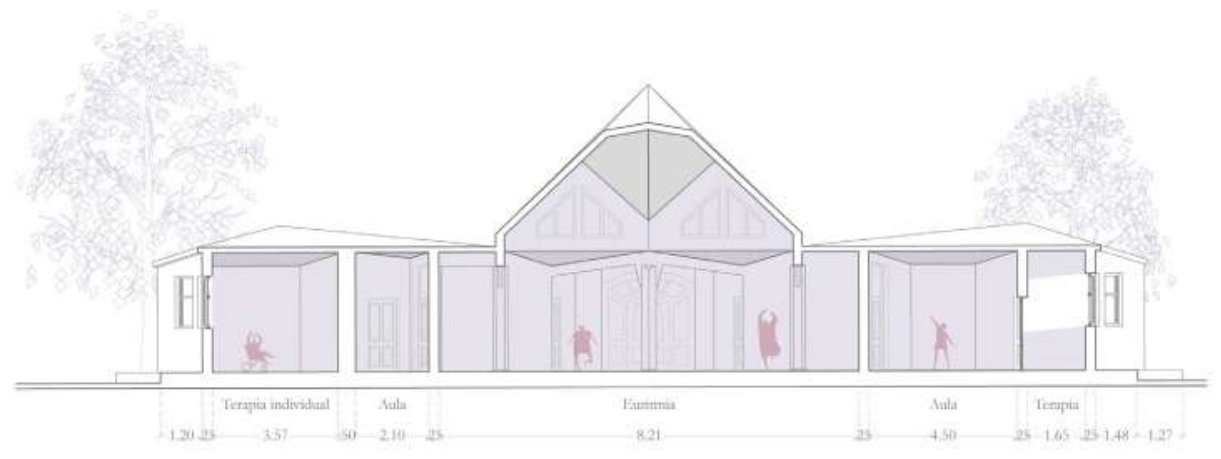

Figura 7: Análisis solar durante verano en el colegio Waldorf-Lima. (Elaboración: Vargas, M. 2017). 


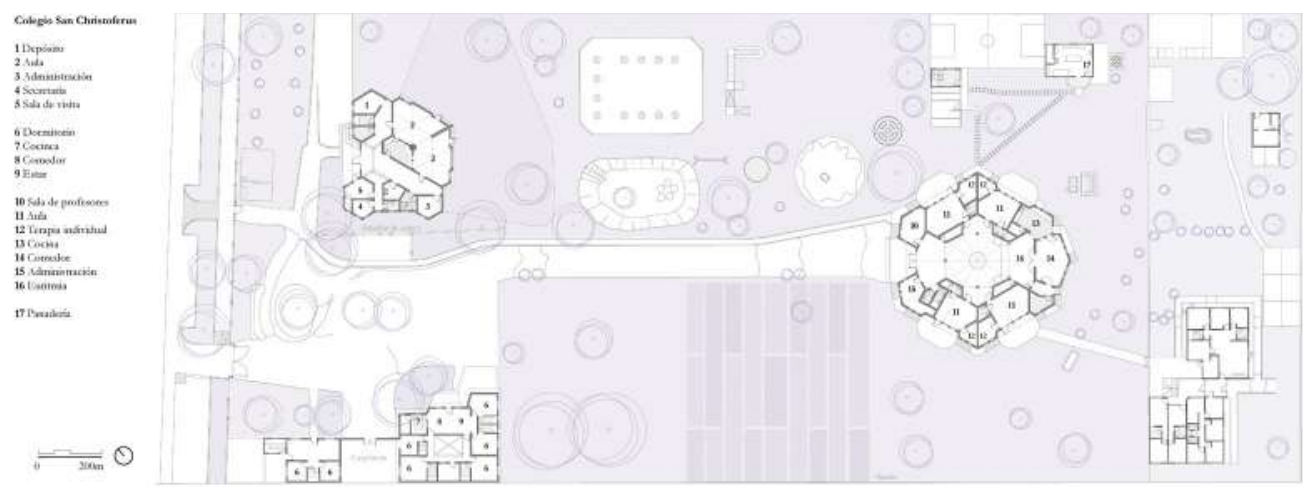

Figura 8: Análisis solar durante verano en el colegio Waldorf-Lima. (Elaboración: Vargas, M. 2017).

gio, así como una vivienda para las personas que vienen de intercambio para ayudar con las labores del colegio. Los dos edificios principales destinados a la enseñanza se conectan a través de un camino que atraviesa la zona de biohuerto y el jardín de juegos (Figura 8).

El segundo pabellón cuenta con cuatro aulas proyectadas como piezas que al ser engranadas unas contra otras proyectan un edificio donde todas las partes responden a un propósito y cada una de estas piezas conectadas conforma un todo que envuelve un espacio central importante para la antroposofía y para la escuela, el espacio de euritmia, convirtiéndose en el corazón del edificio (Figura 8). Así, al igual que la pedagogía Waldorf, el colegio San Christoferus ha sido concebido desde una perspectiva holística, brindando espacios que respondan a los niños que habi$\tan$ a diario dentro de esta arquitectura.

La pieza modular está conformada por un salón, un baño y un espacio especial para terapias individuales. La forma orgánica de las aulas está pensada para que las energías de los niños puedan fluir libremente a través de estas, al tratarse de espacios de enseñanza para niños con habilidades especiales se propuso un espacio de terapia individual en cada salón. Las aulas cuentan con doble acceso tanto del exterior como del interior, tener acceso directo al exterior permite a los niños tener una relación directa con la naturaleza, además en caso de que ocurra algún accidente o alguno de los niños presente un episodio de epilepsia puede ser evacuado hacia el exterior rápidamente sin tener que recorrer todo el edificio, de esta manera se asegura que todas las actividades se realicen dentro del aula.

Para la antroposofía el diálogo constante entre los muros y el todo es fundamental, esto se evidencia en los quiebres que adopta la cubierta y la relación de estos con los muros, donde se da un diálogo constante entre los elementos arquitectónicos. Además, una de las características interesantes del colegio es la sección del techo y el movimiento de este que se puede observar al estar en el interior del salón brindándole una esencia particular a cada uno.

En cuanto al uso de color, el segundo pabellón donde están las cuatro aulas de trabajo ha sido trabajado en tonos amarillos y rojos. Las cuatro aulas que se encuentran en este edificio juegan con las diferentes gamas de colores, partiendo desde un aula que utiliza los tonos rojos-rosados, siguiendo a una donde la tonalidad roja ha sido disminuida cambiando a un naranja, continuando con un salón con tonalidades amarillas y, finalmente, un aula con los muros pintados en color azul, la cual actualmente es utilizada como un espacio para almacenar las piezas de madera trabajadas por los alumnos, maestros, voluntarios y encargado del colegio.

A diferencia de otros colegios Waldorf, donde la teoría del color es utilizada como una forma de controlar el temperamento del niño y 
donde el color va cambiando según el grado de estudio y la edad en la que se encuentran los alumnos, en el caso del colegio San Christoferus, todos los niños conviven en el mismo espacio, los salones se convierten en espacios compartidos por todos, por lo tanto, no tiene un mismo impacto aplicar el concepto del color en un colegio dirigido a niños sin discapacidades que habitarán el espacio durante un horario fijo de horas, que aplicarlo en un colegio donde los niños utilizarán el espacio en menor medida.

\section{Conclusiones}

El análisis, basado en la recolección de datos, entrevistas, levantamientos arquitectónicos y registros fotográficos; ha permitido conocer y aproximar la mirada a la situación de un nuevo modelo de arquitectura educativa que se viene desarrollando en la ciudad de Lima. Se ha estudiado dos colegios que se encuentran insertados en realidades diferentes. Además, ambos casos de estudio han sido concebidos desde un punto de vista antroposófico y estos han marcado un momento en la historia de la educación en el Perú.

La antroposofía adquiere importancia principalmente por el interés puesto en el hombre y por la visión holística que se tiene del este, así, la antroposofía busca responder no solo a necesidades físicas sino también a necesidades anímicas y espirituales, que varían con el tiempo.

La arquitectura antroposófica, se convierte en un elemento importante para el desarrollo del hombre, ya que esta representa la manifestación física de los deseos y sus necesidades. Considera que todos los elementos externos al cuerpo físico influyen y tienen un impacto sobre la percepción del espacio de la persona, el espacio no solo influye por lo que se realiza en el interior de este, sino por lo que le transmite a la persona y por las sensa- ciones que puede tener sobre la persona. Así, el objetivo principal de la arquitectura antroposófica es generar espacios que acompañen a la persona a través de su desarrollo.

La arquitectura educativa antroposófica busca desarrollar espacios que respondan a las necesidades de los niños que habitarán dentro de esta, la forma se considera esencial, pues este tendrá un impacto sobre la percepción del niño, así la forma del espacio contribuye a favor del aprendizaje y crecimiento del niño. La arquitectura educativa antroposófica da una serie de criterio que pueden ser considerados o no al momento de proyectar arquitectura educativa, tales como: no generar espacios rígidos, los ambientes deben ser cálidos y amables, espacios que expresen y permitan la libertad del alma y el cuerpo del niño.

Por otro lado, la relación directa que se da entre arquitectura, medio ambiente y hombre es esencial para la antroposofía, ya que esta considera a estos tres elementos como un todo que se vinculan. La preocupación por utilizar materiales cercanos a la naturaleza, criterios bioclimáticos y el aprovechamiento de los recursos del lugar, recae en la idea de generar un menor impacto en la naturaleza.

La importancia de un sistema educativo con un panorama diferente al de la escuela tradicional en la ciudad de Lima contribuye a transmitir una nueva idea de espacios de aprendizaje que no solo responden a actividades que se realizan en el interior, sino espacios que buscan transmitir una idea de sociedad. Se evidencia entonces que la arquitectura no nace de modelos predeterminados, sino que esta debe responder al niño y al tiempo en la historia en la que se encuentra. 


\section{Referencias}

Bayes, K. (2014). Arquitectura viva. Madrid, España: Rudolf Steiner.

Cabanellas, I., \& Eslava, C. (2005). Territorios de la Infancia, diálogos entre pedagogía y arquitectura. Barcelona, España: Grao.

Carlgren, F. (1989). Pedagogía Waldorf: una educación hacia la libertad. Madrid, España: Editorial Rudolf Steiner.

Clouder, C., Rawson, M. (2002). Educación Waldorf: Ideas de Rudolf Steiner en la práctica. Madrid, España: Editorial Rudolf Steiner.

Grimm, R. (s.f.). Pedagogia Curativa Antroposófica. Recuperado de https:// docplayer.es/79493942-Pedagogia-curativaantroposofica.html

Jiménez, A. (2009). La escuela nueva y los espacios para educar. Educación y Pedagogía, 21(54), 103-125.

Patzlaff, R. (2007). Indicaciones de Pedagogía
Waldorf: para niños de 3 a 9 años. Madrid, España: Rudolf Steiner.

Petrash, J. (2002). Understanding Waldorf Education: Teaching from the Inside Out. New York, USA: Gryphon House.

Quiroga, P. y Girard, O. (2015). La expansión internacional de la pedagogía Waldorf: un análisis histórico. Temps d'Educació. 48(1), 91109.

Steiner, R. (1978) Curso de educación curativa. México D.F.: Antroposófica.

Steiner, R. (1991). La educación del niño desde la antroposofía. Metodologia de la enseñanza y las condiciones vitales de la educación. Madrid, España: Editorial Rudolf Steiner.

Toranzo, V. (2007). ¿Pedagogía vs. Arquitectura? Los espacios diseñados para el movimiento. Buenos Aires, Argentina: s.e. 